\title{
An Improved Computational Technique for Calculating Electromagnetic Forces and Power Absorptions Generated in
Spherical and Deformed Body in
Levitation Melting Devices
}

\author{
Jin-Ho Zong, Julian Szekely, and Elliot Schwartz
}

207072

\begin{abstract}
An improved computational technique for calculating the electromagnetic force field, the power absorption and the deformation of an electromagnetically levitated metal sample is described. The technique is based on the volume integrat method, but represents a substantial refinement; the coordinate transformation employed allows the efticient treatment of a broad class of rotationally symmetrical bodies. Computed results are presented to represent the behavior of levitation melted metal samples in a multi-coil, multi-frequency levitation unit to be used in microgravity experiments. The theoretical predictions are compared with both analytical solutions and with the results of previous computational efforts for the spherical samples and the agreement has been very good. The treatment of problems involving deformed surfaces and actually predicting the deformed shape of the specimens breaks new ground and should be the major usefulness of the proposed method.
\end{abstract}

\section{INTRODUCTION}

W HILE levitation melting has been used quite extensively in metallurgical research [1]. 121. 131, it has recently assumed greatly increased importance as a possible vehicle for space experimentation [4], [5], [6]. Electromagnetic levitation (EML) is attractive for the containerless processing of conducting melts in a microgravity environment because, through the use of a multiple coil arrangement, it is possible to provide for the independent control of the positioning forces and the power employed for heating the sample [7]. A schematic sketch of such an arrangement is given in Fig. 1, where the upper and lower coils from generator i) are used for positioning, the intermediate coils of ii) are used for heating and the central coil iii) may be used for shaping or "squeezing" the sample.

Manuscript received April 19. 1990: revised December 19. 1991. The authors acknowledge the partial supports of finance provided by the Korea Science and Engincering Foundation and by the National Acronatic and Space Administration of the USA.

The authors are with the Deparment of Materials Science and Engineering. Massachusetts Institute of Technology. Cambridge. MA (02139

ItiEt Log Number 9106725 .

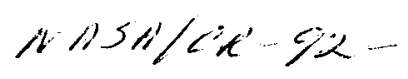

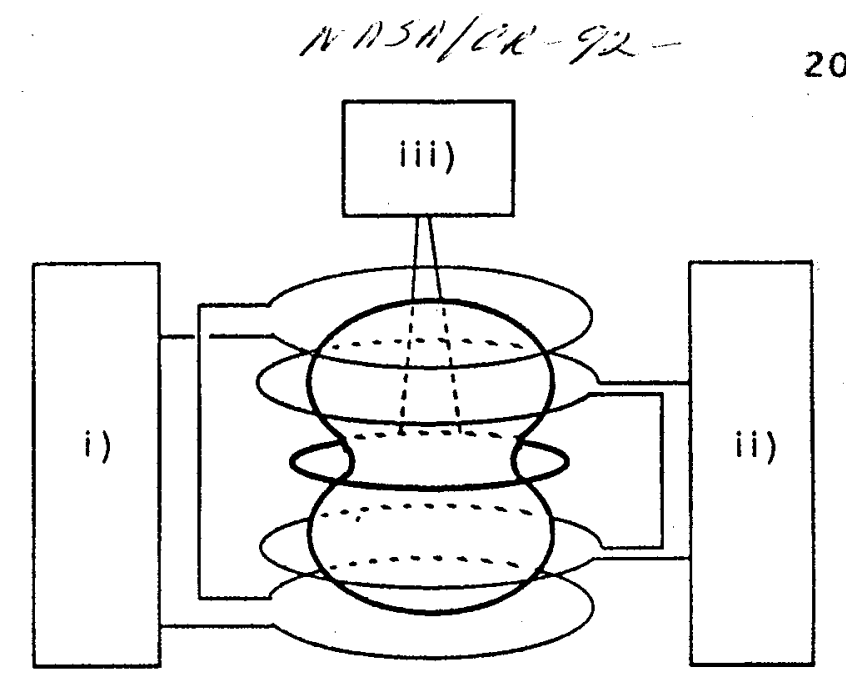

Fig. 1. Schematic view of EML device using multifrequency system: RFGenerator for i) positioning, ii) heating, iii) shaping coil systems.

In the rational design of in-flight experiments it is critical that the lifting (positioning) and shaping forces, as well as the power absorbed by the sample. be known quite accurately. The main parameters of the EML device are geometry of coil and sample, applied current, applied frequency, and conductivity of the sample.

In representing this system we encounter problems at the following levels: 1) calculation of the lifting force and the power absorption by a symmetrically placed spherical sample: 2) calculation of the lifting force and the power absorption by a deformed sample, the shape of which is predetermined; 3 ) calculation of the lifting force and the power absorption for a sample, the shape of which is defined as a result of the calculations; 4) the repeat of the previous case, but with an allowance for fluid flow; and 5) repeat of the previous case but with an allowance for transient behavior. Case 1) may be tackled analytically; indeed the available analytical solutions will serve as an excellent benchmark against which the computed results may be tested. The subsequent cases all need a numerical approach; furthermore, cases 3)-5) will require a highly efficient computational scheme because of the iterative 
procedures that are involved. While the ultimate objective of the research is to tackle cases 1) through 5), in the present paper we shall confine our attention to cases 1)-3).

In a recent paper, G. Lohofer [8] presented an analytical solution for the total power absorption $\bar{P}_{s}$ by a spherical sample placed in a field exhibiting rotational symmetry.

$$
\begin{aligned}
\bar{P}_{s}= & \frac{\pi}{2 R_{s} \sigma_{s}} \sum_{n} \sum_{l=1}^{\infty} H_{l}\left(q_{n}\right)\left(\tilde{I}_{n, l}^{2}+2 \sum_{n^{\prime}>n} \delta_{\omega_{1,}, \omega_{n} \cdot}\right. \\
& \left.\cdot \cos \left(\alpha_{n}-\alpha_{n^{\prime}}\right) \tilde{I}_{n, l} \tilde{I}_{n^{\prime}, l}\right) .
\end{aligned}
$$

where

$$
\begin{aligned}
H_{l}\left(q_{n}\right) & =\sum_{k=1}^{\infty} \frac{8 q_{n}^{4}}{4 q_{n}^{4}+x_{l+1 / 2 . k}^{4}} \\
q_{n} & =\frac{R_{s}}{d_{n}} \\
I_{n . l} & =\sqrt{\frac{2 l+1}{l(l+1)}} I_{n}\left(\frac{R_{s}}{R_{n}}\right)^{\prime} \sin \theta_{n} P_{l}^{\prime}\left(\cos \theta_{n}\right) .
\end{aligned}
$$

$I_{n} \quad$ applied current in coil $n$

$x \quad$ zeroes of spherical Bessel function

$P \quad$ associated Legendre polynomial

$R_{s}, R_{n}$ radius of sample and coil

$\theta_{n} \quad$ angle of coil position from the rotational axis

$\sigma_{s} \quad$ conductivity of sample

$\omega \quad$ angular frequency

$n, n^{\prime} \quad$ index of coils

$\delta \quad$ Kronecker delta

$d_{n} \quad$ skin depth

$\alpha \quad$ phase

$l, k$ summation index.

However, as noted before. this analytical result has some limitations as a potential design tool because it is confined to spherical samples, while in reality the samples may deform; furthermore, the analytical results do not provide information on the local values of the induced force field or heat generation. The local values of the field would, of course, be needed for calculating or even estimating melt circulation. Therefore, the numerical calculation of the heating rate and of the induced electromagnetic forces is highly desirable.

Several standard techniques are available for calculating the induced current and force field produced in a metallic sample surrounded by induction coils. These include volume integral method (VIM) [9], [10], finite element method (FEM) [11], [12], and boundary element method (BEM) [13], [14]. Each of these techniques has advantages and drawbacks.

One of the practical difficulties in performing these numerical calculations by FEM or VIM techniques is associated with the fact that the very small skin depth resulting from the high-frequency coil current might cause numer- ical errors because the induced magnetic field and associated parameters decrease exponentially within the skin depth [13]. Therefore, care has to be taken that a sufficiently large number of grid points are placed within the skin depth region, which, in turn, requires substantial computing time. One alternative is the BEM, which could be especially attractive for very small skin depths.

However, a typical EML tends to utilize a very broad range of frequencies and currents, in the range of $10^{2}-$ $10^{3} \mathrm{kHz}$ and $10^{2} \sim 10^{3} \mathrm{~A}$, in order to obtain sufficient Joule heating and positioning or deformation. Furthermore, in a typical application, as the sample is being heated or is allowed to cool, major changes will occur in temperature, and hence in the electrical conductivity and the skin depth. In the EML experiments currently contemplated, the ratio of skin depth/sample radius can range from about $1 / 2$ to $1 / 30$. It follows that the domain of interest may not be necessarily confined to the near surface region.

The volume integral (coupled circuit or mutual inductance) method has the advantage that it is computationally very efficient and simple to use. However, in its original form, it has the drawback that the elements have a rectangular cross-section and curved surfaces are approximated in a staircase fashion which can introduce significant errors and place major demands on the computing time in tackling free surface problems [15] [16]. Previously reported studies for levitation melting based on the mutual inductance method have postulated a very small skin depth, and hence plane wave approximation. and used only one grid point on the surface in order to alovid this difficulty $[17][18]$. Since the induced magnetic flux density and force depend not only on the magnitude but on the spatial variation of the induced current in the region near the surface, this approach may not be always appropriate, especially when high accuracy is needed, e.g., in microgravity processing. Furthermore, as the forces induced within the skin depth form the driving force for fluid motion within the sample as well as for the hape change. they have to be known quite accuratcl!

It follows from the foregoing that while there are many possible computational schemes available for representing the interaction of a sample and the surrounding coils, the existing volume integral methods are not optimally suited to levitation melting because of the extreme case of high curvature and broad range of skin depths that halle to be tackled. These problems would be brought into focus even more sharply when the behavior of deformed samples is being examined. This has motivated the development of the computational technique to be described in the following.

Subsequently, we shall present a numerical scheme based on the volume integral method, applicable to axisymmetric deformed samples as well as spheres in the levitation melting and positioning device, which could minimize the numerical errors that were inherently associated with the previously used computational schemes. We shall also show how this technique can be effectively inter- 
linked with the shape determination algorithm for calculating the shape of the electromagnetically deformed droplets in EML devices.

\section{Formulation}

\section{A. An Improved Volume Integral Technique for Electromagnetic Calculation}

In the numerical scheme the electromagnetic levitating forces and Joule heating are obtained in a rotationally symmetric system of otherwise arbitrary shape by a volume integral method through manipulating the wellknown concept of mutual inductances. Let us consider an axisymmetric system which is discretized into $N$ elementary circuits in the sample and a total of $\Sigma_{f=1}^{F} M_{f}$ external coils of $F$ different frequency systems as shown in Fig. 2 .

The vector potential $\vec{A}$ (defined by $\vec{B}=\nabla \times \vec{A}$ ) at the position $\vec{r}$ is readily written by Biot-Savart's law. In the absence of ferromagnetic materials the current flows in the $\phi$-direction alone.

$$
\vec{A}(\vec{r})=\frac{\mu_{0}}{4 \pi} \int_{\text {vol }} \frac{\vec{J}\left(\vec{r}^{\prime}\right)}{\left|\vec{r}-\vec{r}^{\prime}\right|} d \nu\left(\vec{r}^{\prime}\right) .
$$

The $\phi$-component of the $i$ th element in the sample is expressed as following;

$$
\begin{aligned}
A_{i}^{(\phi)}= & \frac{\mu_{0}}{4 \pi}\left[\sum _ { f = 1 } ^ { F } \left\{\sum_{j=1}^{N} \int\left(J_{f}\right)_{j} d S_{j} \frac{d l_{j}}{r_{i j}}\right.\right. \\
& \left.\left.+\sum_{k_{f}=1}^{M_{f}} \int \frac{\left(I_{f}\right)_{k_{f}} d l_{k_{f}}}{r_{i k_{f}}}\right\}\right],
\end{aligned}
$$

where the first term on the right-hand side represents the induced potential by other elementary circuits and itself while the second term is due to the external source coils, $\mu_{0}$ is the magnetic permeability, and

$d S \equiv$ cross sectional area of the circuit

$r_{i j}, r_{i k f} \equiv$ distance between coil- $i$ and coil $j$ or $k$

$d l \equiv$ line element of circuit in $\phi$-direction

$J_{f}, I_{f} \equiv$ induced current density in the sample, and applied current in the coil (a $180^{\circ}$ phase difference in time is expected between them) which have general complex forms containing the coil frequency and phase in each of the systems $\omega_{f}, \alpha_{f}$ :

On expressing the current density, etc. using phasor notation we have

$$
J_{f}=J_{0} \exp j\left(\omega_{f} t-\alpha_{f}\right)=J_{f}^{R}+j J_{f}^{\prime} \ldots
$$

where superscripts $R$ and $I$ denote the real and the imaginary part, and

$$
J_{f}=\sigma E=-\sigma \frac{d A_{f}}{d t}
$$

Using (4), (5) the following relations are obtained

$$
\begin{aligned}
& A_{f}^{R}=-\frac{1}{\sigma \omega_{f}} J_{f}^{l} \\
& A_{f}^{l}=\frac{1}{\sigma \omega_{f}} J_{f}^{R} .
\end{aligned}
$$
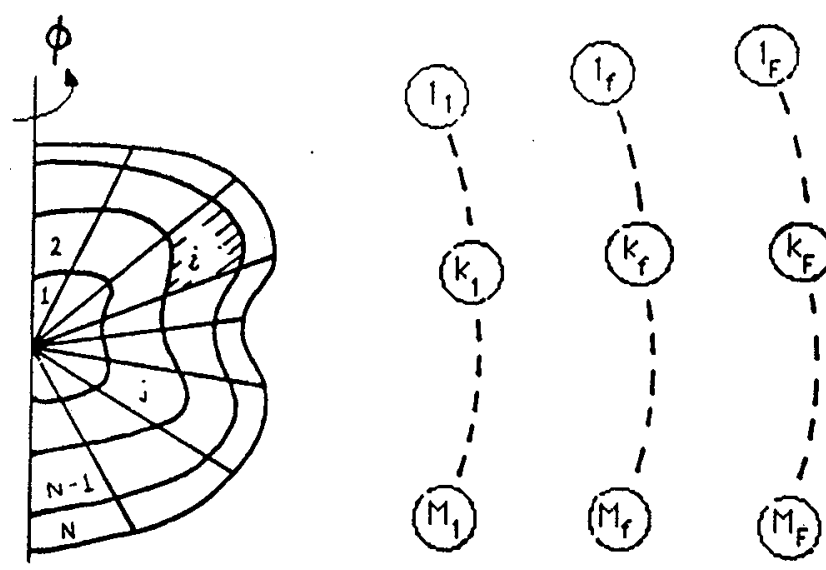

Fig. 2. Discretization into rotational elementary circuits on the physical domain (PD) in multifrequency system.

Substituting (3) into (6) and multiplying by $\oint \cdot d \vec{l}_{i}$ we obtain

$$
\begin{gathered}
\sum_{f=1}^{F} \oint\left(J_{f}\right)_{i}^{l} \cdot d \vec{l}_{i}+\sigma \sum_{f=1}^{F} \omega_{f} \sum_{j=1}^{N} M_{i j}\left(J_{f}^{R} \cdot S\right)_{j} \\
=-\sigma \sum_{f=1}^{F} \omega_{f} \sum_{k f=1}^{M_{f}} M_{i k_{j}}\left(l_{f}\right)_{k_{f}}^{R}, \quad i=1, N \\
\sum_{f=1}^{F} \oint\left(J_{f}\right)_{i}^{R} \cdot d \vec{l}_{i}-\sigma \sum_{f=1}^{F} \omega_{f} \sum_{j=1}^{N} M_{i k_{f}}\left(J_{f}^{l} \cdot S\right)_{j} \\
=+\sigma \sum_{f=1}^{F} \omega_{f} \sum_{k f=1}^{M_{f}} M_{k_{f}}\left(I_{f}\right)_{k_{f}}^{l}, \quad i=1, N,
\end{gathered}
$$

where the mutual inductance between coil $i$ and $j$ or $i$ and $k_{f}$ can be obtained by the elliptic integral in the $\phi$-direction:

$$
\begin{aligned}
M_{i j} & =\frac{\mu_{0}}{4 \pi} \oint \frac{d \vec{l}_{j}}{r_{i j}} \oint d \vec{l}_{i}, \\
M_{i k_{f}} & =\frac{\mu_{0}}{4 \pi} \oint \frac{d \vec{l}_{k f}}{r_{k f}} \oint d \vec{l}_{i} .
\end{aligned}
$$

Dividing (7) and (8) by $\sigma$ and recalling

$$
R_{i}=\frac{\oint d \vec{l}_{i}}{d S_{i} \sigma}
$$

and

$$
J_{i}=\frac{I_{i}}{d S_{i}}
$$

we have the following relationships

$$
\begin{gathered}
\sum_{f=1}^{F}\left(I_{f}\right)_{i}^{\prime} R_{i}+\sum_{f=1}^{F} \omega_{f} \sum_{j=1}^{N} M_{i j}\left(I_{f}\right)_{j}^{R}=-\sum_{f=1}^{F} \omega_{f} \Phi_{f}^{R} \\
\sum_{f=1}^{F}\left(I_{f}\right)_{i}^{R} R_{i}-\sum_{f=1}^{F} \omega_{f} \sum_{j=1}^{N} M_{i j}\left(I_{f}\right)_{j}^{\prime}=+\sum_{f=1}^{F} \omega_{f} \Phi_{f}^{I},
\end{gathered}
$$


where, $\Phi$ is the flux in the source terms, which is determined by applied current and the geometric relationships

$$
\begin{aligned}
& \Phi_{f}^{R}=\sum_{k f=1}^{M_{f}} M_{i k f} I_{k f}^{R} \\
& \Phi_{f}^{\prime}=\sum_{k_{f}=1}^{M_{f}} M_{i k_{f}} I_{k f}^{\prime}
\end{aligned}
$$

By eliminating $\left(l_{j}\right)_{j}^{\prime}$ from (13) and (14);

$$
\begin{array}{r}
\sum_{f=1}^{F}\left(R_{i}+\omega_{f}^{2} \sum_{j=1}^{N} \frac{M_{i j}}{R_{j}} \sum_{i=1}^{N} M_{i j}\right)\left(I_{f}\right)_{i}^{R} \\
=\sum_{f=1}^{F} \omega_{f}\left(\Phi_{f}^{l}-\omega_{f} \sum_{j=1}^{N} \frac{M_{i j}}{R_{i}} \Phi_{l}^{R}\right),
\end{array}
$$

which may be expressed as the following system of linear equations

$$
[R+X] I=C \quad f=1, F
$$

where

$$
\begin{aligned}
& R \text { diagonal matrix of resistances of the circuit-ele- } \\
& \text { ments } \\
& X \text { full matrix containing the self and mutual induc- } \\
& \text { tance } \\
& I \text { vector of the unknown currents } \\
& C \text { vector of applied sources. }
\end{aligned}
$$

For applied currents of a given frequency, the system of linear equations (18) can be solved simultaneously using matrix inversion methods. The induced current density, $J^{R}$ and $J^{\prime}$, and hence $\vec{A}$ are obtained by (13), (12), and (6). With $J$ and $\vec{A}$ known, the magnetic flux density $(\vec{B})$ can be obtained either by Biot-Savart's law or by the direct differentiation of the vector potential $(\nabla \times \vec{A})$. For multiple frequencies, the total $J, \vec{A}$. and $\vec{B}$ are summed from their corresponding components calculated for wach frequency. The magnetic field and induced current field having been so calculated, the time-averaged levitation force $\left(F_{l}\right)$ and the power absorption of the sample $\left(\bar{P}_{s}\right)$ and are given by the following expressions:

$$
\begin{aligned}
& F_{l}=\frac{1}{T} \int_{0}^{T} \int_{\text {vol }} \frac{1}{2} \operatorname{Re}\left\{J \times B^{*}\right\} d v d t \\
& \bar{P}_{s}=\frac{1}{T} \int_{0}^{T} \int_{\text {vol }} \frac{1}{2} \operatorname{Re}\left\{\frac{J \cdot J^{*}}{\sigma}\right\} d v d t .
\end{aligned}
$$

To perform the calculations a grid structure has to be defined. As noted in the introduction we seek to establish a flexible gridding system appropriate for an axisymmetric body of otherwise arbitrary shape. The grids are generated in a rectangular uniform calculation domain (CD2) and can be transformed into the physical domain (PD) through the intermediate calculation domain (CDI) as shown in Fig. 3,

$$
\begin{aligned}
& r=f(\bar{r}, h(\theta)) \quad \bar{r}=F(r, h(\theta)) \\
& \theta=g(\bar{\theta}) \quad \bar{\theta}=G(\theta) .
\end{aligned}
$$

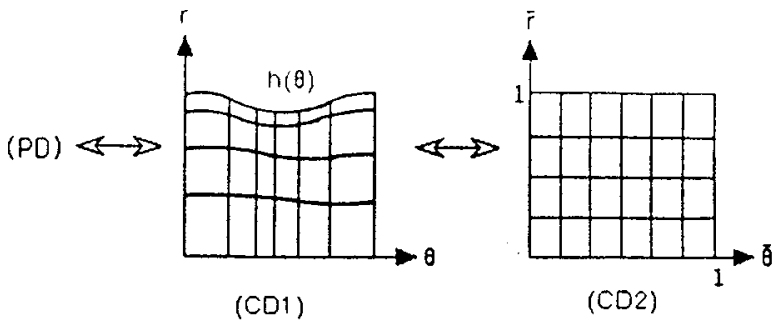

Fig. 3. Schenatic representation of coordinate transformation.

Two kinds of control functions were used for grid generation. The contraction/stretching functions $f$ and $g$ are used for grid control, $F$ and $G$ are their inverses, and $h(\theta)$ is the shape function of the sample. The function $f(r)$ is used from the general transformation family proposed by Roberts $|19|$. The function $h(\theta)$ can be given analytically or numerically. The spherical shape is the special case of $h(\theta)=$ constant .

The transformation from CD2 to CD1 can be done by writing:

$$
\begin{aligned}
& \frac{\partial}{\partial r}=\frac{\partial \bar{r}}{\partial r} \frac{\partial}{\partial \bar{r}}+\frac{\partial \bar{\theta}}{\partial r} \frac{\partial}{\partial \bar{\theta}}=\frac{\partial F}{\partial r} \frac{\partial}{\partial \bar{r}} \\
& \frac{\partial}{\partial \theta}=\frac{\partial \bar{r}}{\partial h} \frac{\partial h}{\partial \bar{\theta}} \frac{\partial \bar{\theta}}{\partial \theta} \frac{\partial}{\partial \bar{r}}+\frac{\partial \bar{\theta}}{\partial \theta} \frac{\partial}{\partial \bar{\theta}}=\frac{\partial F}{\partial h} \frac{\partial h}{\partial \bar{\theta}} \frac{\partial G}{\partial \theta} \frac{\partial}{\partial \bar{r}}+\frac{\partial G}{\partial \theta} \frac{\partial}{\partial \bar{\theta}}
\end{aligned}
$$

and the transformation from CDI to PD, in other words. from spherical coordinates $(r, \theta, \phi)$ to Cartesian coordinates $(x, y, z)$, is given by the general geometric retationship between two coordinate systems $[20]$. For example. the area element $(d S)$ and the magnetic field density are expressed as follows:

$$
\begin{aligned}
d S^{(\phi)}= & \left|\frac{\partial \vec{r}}{\partial r} \times \frac{\partial \vec{r}}{\partial \theta}\right| d r d \theta \\
= & r \frac{G^{\prime}(\theta)}{F^{\prime}(r)} d \vec{r} d \bar{\theta}(-i) \\
\vec{B}= & \nabla \times \vec{A} \\
= & \mid \frac{1}{f(\bar{r}, h(\theta))^{2} \sin \theta}\left\{\frac{\partial F}{\partial h} \frac{\partial h}{\partial \bar{\theta}} \frac{\partial G}{\partial \theta} \frac{\partial}{\partial \bar{r}}+\frac{\partial G}{\partial \theta} \frac{\partial}{\partial \bar{\theta}}\right\} \\
& \cdot\left(A_{\phi} f(\bar{r}, h(\theta)) \sin \theta\right) \mid \hat{r} \\
& \quad+\left[-\frac{F^{\prime}(r, h(\theta))}{f(\bar{r}, h(\theta))} \frac{\partial}{\partial \bar{r}}\left\{f(\bar{r}, h(\theta)) A_{\phi}\right\}\right] \hat{\theta} .
\end{aligned}
$$

\section{B. Application to Shape Determination of Lavitated Droplet}

The deformed shape of the levitated droplet was calculated using the local force balance at the surface of the sample in conjunction with an iterative technique [21] [22]. The instantaneous displacement of a surface element 
was derived from the surface force balance with a constant-volume constraint which gave the new shape until the normal pressures reached equilibrium. The actual algorithm used was as follows (see flowchart in Appendix).

1) The magnetic field strength is calculated for an initial shape according to the previously described scheme. 2) By assuming an infinitesimal displacement $\Delta \vec{x}$ in the normal direction of magnitude proportional to the local residual force, the force balance at the surface is given by

$$
\vec{f}_{n}=-\left\{\rho g z+\frac{B_{s}^{2}}{2 \mu}+\gamma\left(K-K_{0}\right)+\lambda\right\} \Delta \hat{n} \propto \Delta \vec{x},
$$

where $\rho$ is density of the sample, $g$ is gravity, $z$ is the vertical distance from the top, $\gamma$ is the surface tension between the liquid metal and the gas, and $\lambda$ is a Lagrange multiplier which is determined by

$$
\lambda=\frac{-\int_{\text {is }}\left\{\rho g z+\frac{B^{2}}{2 \mu}+\gamma\left(K-K_{0}\right)\right\} d S}{\int_{\partial \Omega} d S} .
$$

$B_{s}$ is the tangential magnetic field at the surface and $K$ is the sum of the local principal surface curvatures, which can be expressed in spherical coordinates by

$$
\begin{aligned}
K= & K_{1}+K_{2}=\frac{r(\theta) r^{\prime \prime}(\theta)-2 r^{\prime}(\theta)^{2}-r(\theta)^{2}}{\left[r^{\prime}(\theta)^{2}+r(\theta)^{2}\right]^{3 / 2}} \\
& +\frac{1-\frac{r^{\prime}(\theta)}{r(\theta)} \cot (\theta)}{\left[r^{\prime}(\theta)^{2}+r(\theta)^{2}\right]^{1 / 2}} .
\end{aligned}
$$

If $K_{0}$ is the curvature of the bottom of the sample where the magnetic pressure is zero then

$$
\begin{aligned}
\Delta \vec{x} & \propto-\left\{\rho g z+\frac{B_{s}^{2}}{2 \mu}+\gamma\left(K-K_{0}\right)+\lambda\right\} \Delta \hat{n} \\
& =-k\left\{\rho g z+\frac{B_{s}^{2}}{2 \mu}+\gamma\left(K-K_{0}\right)+\lambda\right\} \hat{r}
\end{aligned}
$$

where $k$ is a constant of proportionality, and $\Delta / \hat{l}$ and $\hat{r}$ are unit vectors in the normal and $r$ directions, respectively.

3) Therefore, the new shape is determined by

$$
r(\theta)=r^{0}(\theta)-k\left\{\rho g z+\frac{B_{s}^{2}}{2 \mu}+\gamma\left(K-K_{0}\right)+\lambda\right\}
$$

4) The electromagnetic forces for the new shape are calculated.

5) The criterion on residual forces on surface points $\left|\vec{f}_{n}\right|<\epsilon$ is checked. If it is not satisfied, return to step 2) and calculate again.

\section{REsultis and DIScussion}

The main features of the improved calculation scheme are the following: 1) Arbitrary axi-symmetric coil config- urations may be accommodated. 2) Multifrequency and multiphase coil currents may be represented. 3) The whole range of skin depths may be tackled. 4) An arbitrary axisymmetric shape of a levitated body can be treated. 5) The computational scheme can be used to predict the shape of the levitated specimen, even for significant deformation.

For the computational methodology of the induced current density distribution, the linear system, as stated in (18), is a symmetric and strictly diagonally dominant matrix because the order of resistances of the circuits is much higher than that of the inductances. It was, therefore, defined as a positive definite matrix and the computations were carried out by the Cholesky decomposition method which could minimize the arithmetic operations. Furthermore, the numerical experience of this study has shown that relatively coarse grids can be used even for the irregular shapes as a result of using the coordinate transformation. Although the drawback of the volume integral method has been known to be that the computing time increases very rapidly with the grid size for solving the linear system (16), the calculation was carried out very efficiently by the algorithm presented above. For the computed results presented below, a $20 \times 20$ mesh was the finest grid used and the cpu time of a set of calculations, including the calculation of the lifting force, power absorption rate and the distribution of the time-averaged local Lorentz force, was about $8 \mathrm{~min}$ on a micro-VAX station 3100 , while $66 \mathrm{~s}$ were required for an $15 \times 15 \mathrm{grid}$ and 26 seconds for an $8 \times 15$ grid.

Fig. 4 shows the computed heat absorption by a spherical beryllium sample as a function of the applied current in the external coils. The calculation was carried out with the same geometry as that described in a previous paper 14], in which the frequency was $107 \mathrm{kHz}$ and the ratio of skin depth to the sample radius was 0.337 . The agreement with G. Lohofer's analytical solution as shown in (1) is seen to be very good in spite of the fact that coarse grids were used.

It is seen that the calculation is readily carried out when the dimension of the skin depth is comparable to the radius of the sample. This is to be expected. However. one may anticipate computational difficulties at higher frequencies and for samples having a higher electrical conductivity.

Fig. 5(a) shows a set of computed results depicting the power absorption as a function of the applied frequency with the grid size as a parameter for a spherical beryllium sample with the same coil geometry as that described in previous paper $[4]$. The results given by the analytical solution are also shown for the sake of comparison. The sensitivity of the computed results to the number of grid points used is clearly seen; on using a $20 \times 20$ grid scheme the accuracy of the numerical predictions is still quite satisfactory. A $20 \times 20$ grid for a very slightly deformed sample is shown in Fig. 5(b)

The actual error is shown explicitly in Fig. 6(a) as a function of the number of grid points used within the skin 


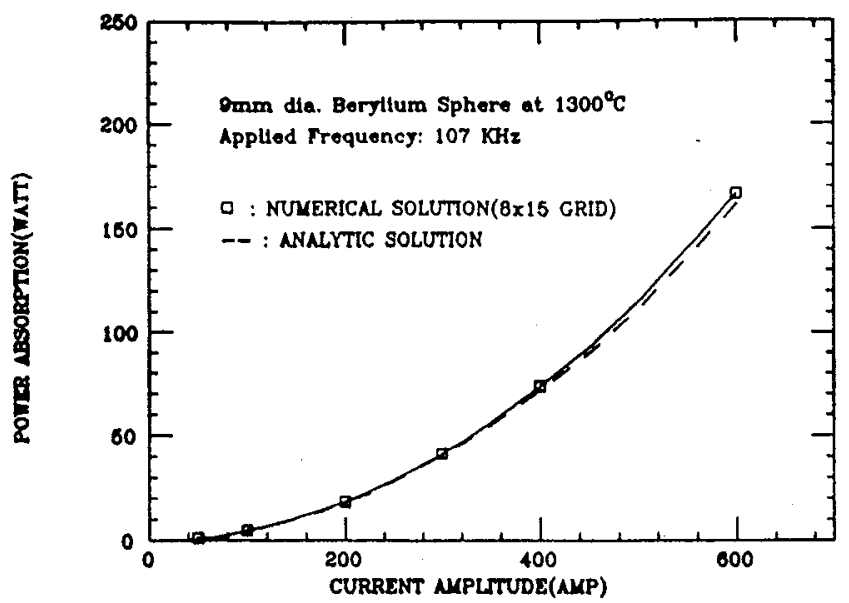

Fig. 4. Variation of power absorption with applied current (by the coils of [4]).

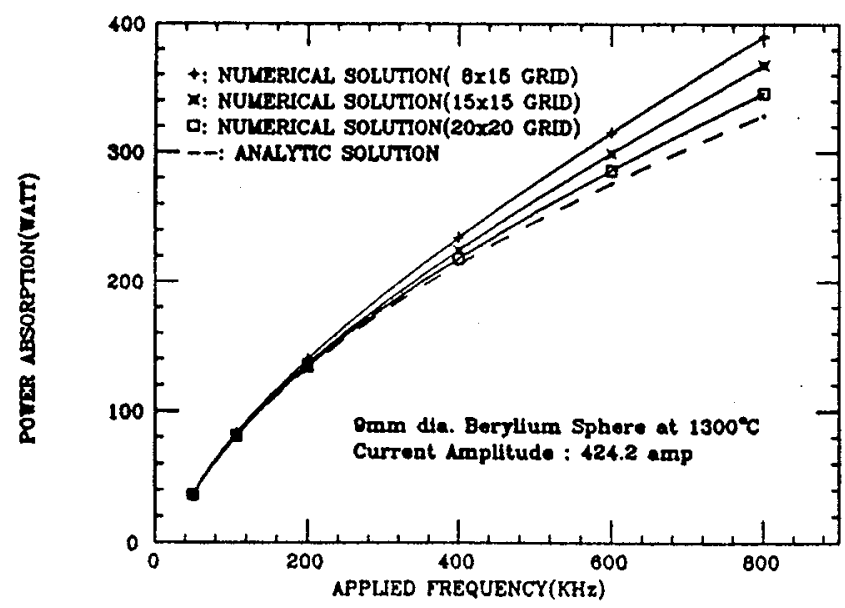

(a)

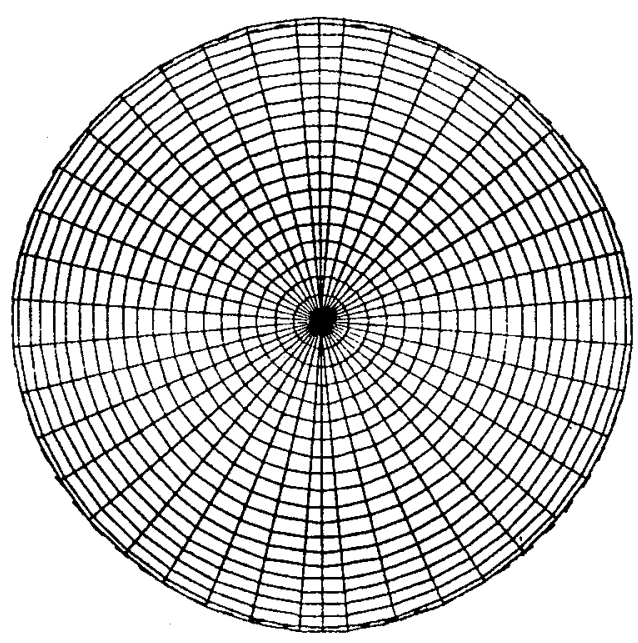

(b)

Fig. 5. (a) Effect of grid size and frequency on the power absorption (by the coils of [4]), (b) $20 \times 20$ grid for very slightly deformed sample.

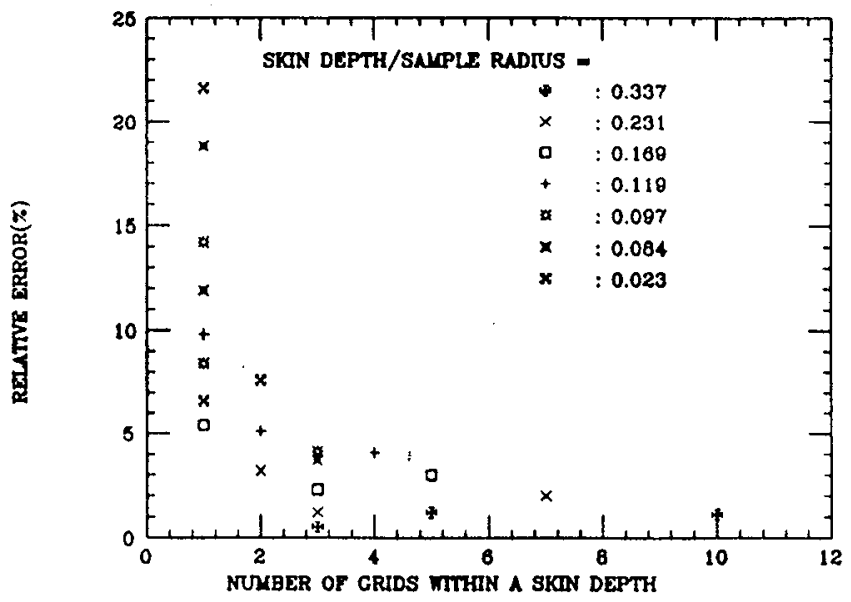

(a)

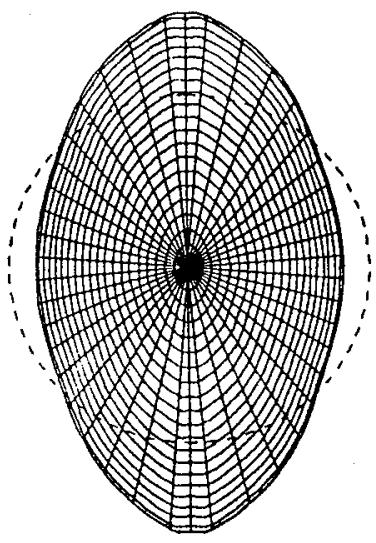

(b)

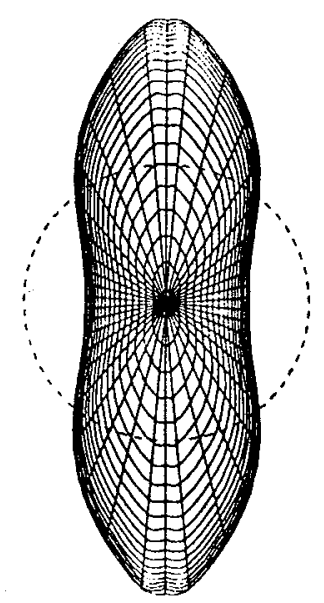

(c)

Fig. 6. (a) Variation of relative error with the number of grid points within one skin depth from the surface. (b) $20 \times 20$ grid with uniformly distributed grid points for highly deformed sample. (c) $20 \times 20$ grid with exponentially distributed grid points for highly deformed sample.

depth. The total number of grid points $(8-20$ in $r$-direction) and the distribution method (uniform, nonuniform or exponential distribution) are tested. It should be remarked that the error is determined not only by the number of grid points located within the skin depth, but by 
the actual location of these grid points. which means that using fewer grid points and a proper distribution is preferable to using a larger number of grid points and a uniform distribution. One may state generally, however, that by placing about $3-4$ grid points within the skin depth one may reduce the error to below $5 \%$. Our experience has shown that the error may be minimized if the location of the grid points follows that same exponential function as does the decay of the induced current and the magnetic flux density as we proceed from the outer surface toward the center of the sample. $20 \times 20$ grids for highly deformed samples with uniformly distributed and exponentially distributed grid points are shown in Fig. 6(b) and (c), respectively.

Fig. 7 illustrates the effect of the sample temperature on the accuracy of the power absorption calculations for the TEMPUS heating coil system. (The schematic cutthrough of the TEMPUS coil system used in this study is shown in Fig. 8 [23]). In the planning of microgravity experiments it is very important to attain specified sample heating rates. The prediction and control of these heating rates is complicated by the strong temperature dependence of the electrical conductivity.

It is seen that the electrical conductivity of the copper sample decreases very markedly with temperature in the solid state, while the conductivity decreases slightly with temperature in the molten state. The corresponding power absorption, however, increases with temperature almost linearly, but shows an abrupt discontinuity near the phase change. which could cause disturbances during melting. The figure shows that by using an exponential grid spacing an excellent agreement may be produced between the numerical calculations and the analytical results.

In designing the electromagnetic levitation device two complementary objectives are sought: 1) to actually lift, i.e., "levitate the sample" in a stable position and 2) to heat the sample.

In the less critical earthbound applications both these objectives may be met by a single-frequency operation, using just one set of coils. In a microgravity environment, where overall efficiency is a major consideration, it has been found that a two-coil arrangement, sketched in Fig. 1 , could provide an improved overall efficiency. As illustrated, in coil i), the rotational direction of the upper coil is directly opposite that in the lower coil, thus creating currents with a phase difference of $180^{\circ}$. Conversely, the coil system in ii) consists of two coils with the same turning direction. The net result of this arrangement is that system i) creates a magnetic quadrupole field, whereas system ii) creates a magnetic dipole field.

Fig. 9, representing magnetic field calculations carried out with the same geometry described in a previous paper [24] with a current of $300 \mathrm{~A}$ and a frequency of $450 \mathrm{kHz}$, shows that the magnetic flux density induced by the dipole field is much larger than that induced by the quadrupole field because of the absence of a current cancellation effect. However, this effect causes the quadrupole field to be stable. This is the reason why a quadrupole arrange-

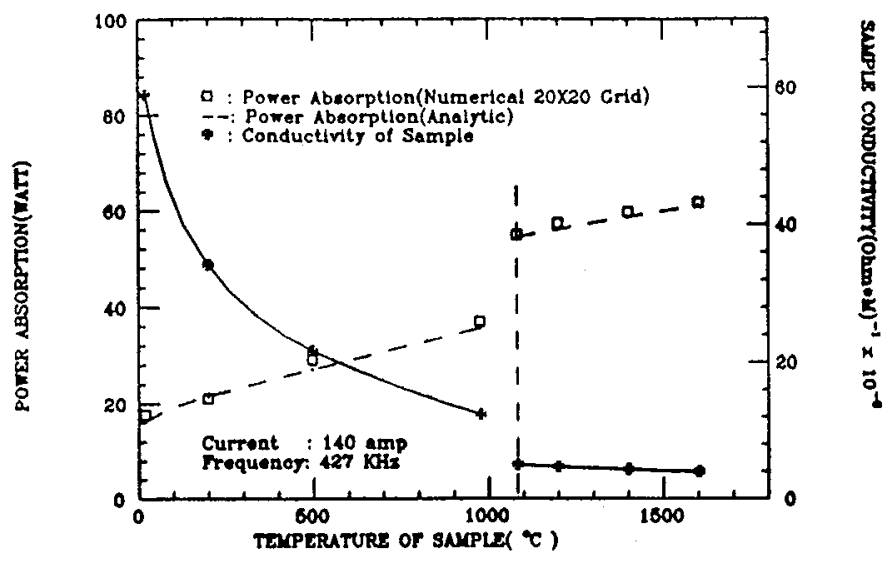

Fig. 7. Temperature dependency of sample conductivity and corresponding power absorption $(10 \mathrm{~mm}$ dia. copper sphere in TEMPUS heating facility).

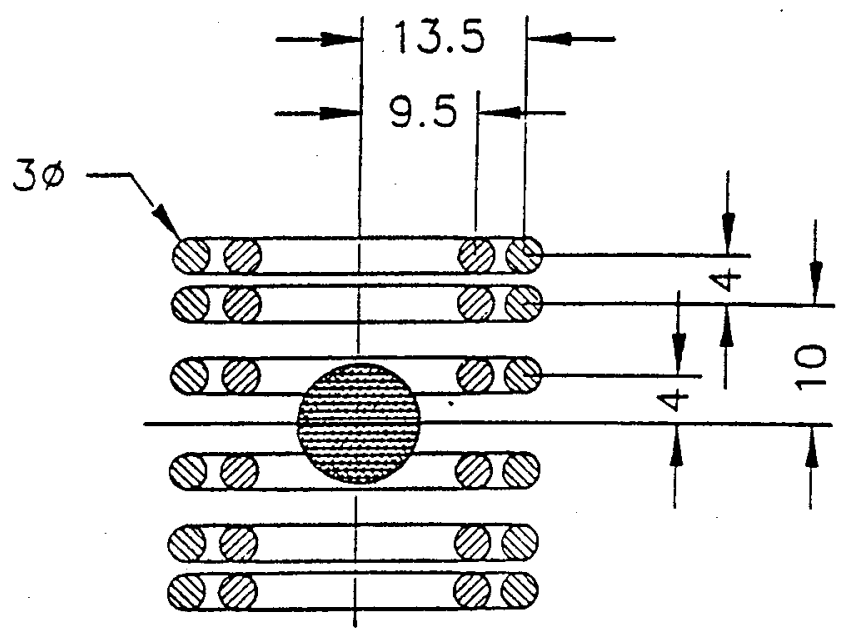

Fig. 8. Schematic cut-through of TEMPUS coil geometry ${ }^{23}$, unit is $\mathrm{mm}$ (inner four coils: dipole field for heating, outer eight coils: quadrupole field for positioning).

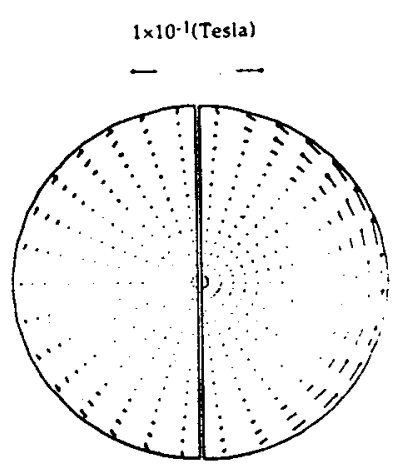

Fig. 9. Typical computed magnetic field density by the quadrupole (left) and dipole (right) field at same condition (for $1 \mathrm{~g}$ iron drop with $300 \mathrm{~A}$, $450 \mathrm{kHz}$. by the coils of $(24))$.

ment is ideal to provide stable lifting, while a dipole arrangement is desirable for heating.

Fig. 10 illustrates the effect of sample position along the vertical axis on the power absorption for a one gram iron drop in the positioning coil system used in the previous paper [24]. Inspection of the figure shows quite good 


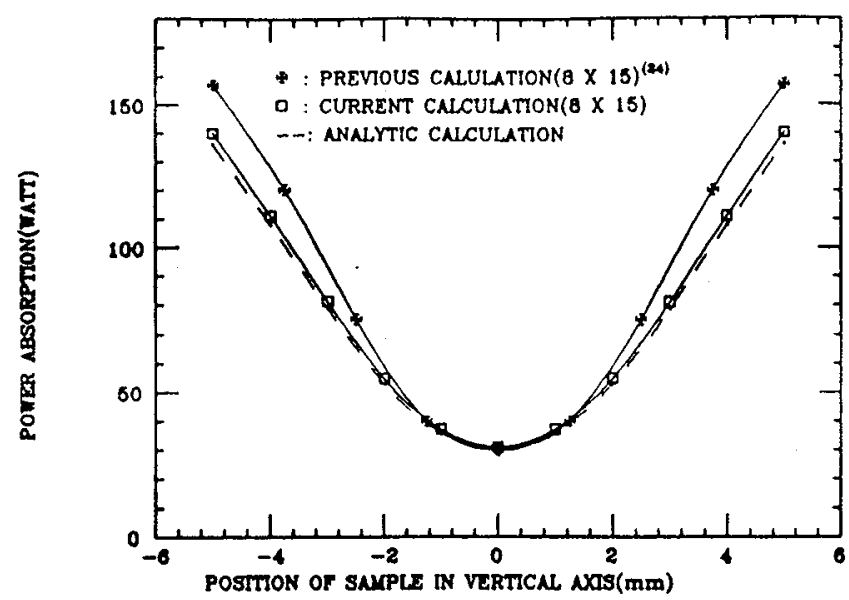

Fig. 10. Comparison of heat-absorption with previous data according of the sample positions (for $1 \mathrm{~g}$ iron drop with $300 \mathrm{~A}$. $4.50 \mathrm{kHz}$. by the coils of $(24)$.

agreement between these three sets of data, computations that were previously performed, analytical predictions, and the results of the present calculations. The present results are in better agreement with the analytical prediction. The figure also suggests that the level of power absorption can be controlled by varying the equilibrium position under earthbound conditions. The minimum value of power absorption occurs when the sample is located at the center of the coil system, but this is not a characteristic of the magnetic quadrupole field.

Fig. 11 shows the effect of the distance between the upper and lower coils ( $d$ in Fig. 11 is half of this distance) of the heating coil system on the power absorption, examined with the same coil geometry as in the previous paper [24] with an in-phase current of $300 \mathrm{~A}$ and a frequency of $450 \mathrm{kHz}$. The closer the two coils, the larger is the heating effect. However, it is important to obtain a uniform heating field which is independent of the sample position in order to have stable experimental conditions. The gradual change in the convexity of the curves indicates that it would be possible to determine the optimal coil design that will create a uniform temperature field regardless of sample position.

The computational scheme allows us to examine the interaction of the "heating" and the "positioning" coils. Fig. 12 illustrates the superpositioning effect of the different frequencies in the heating and positioning coil systems. When the levitated sample is located at an equal distance from the two coils along the rotational axis of symmetry, the maximum power absorption is produced by the heating coil system and the minimum power absorption is produced by the positioning coil system. However, by the proper combination of the two coil systems. uniform heating capability can be obtained which is independent of the sample position along the axis of symmetry. As described above, the positioning coil generates a large stable force field, while the heating coil creates an unstable force. Fig. 13 shows that the superposition of the two coil systems produces a stable force field, which im-

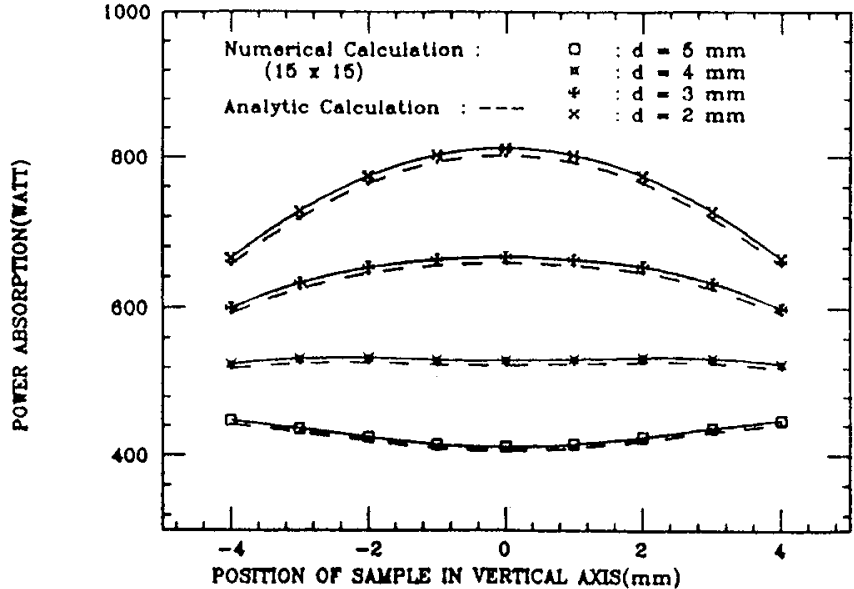

Fig. 11. Fifects of coil separation and the sample positions on the power absarption for $1 \mathrm{~g}$ iron drop with in-phase 300 A current $\$ .50 \mathrm{kH}$; by the coils of 1241 , d; hall distance between upper and lower coils\}.

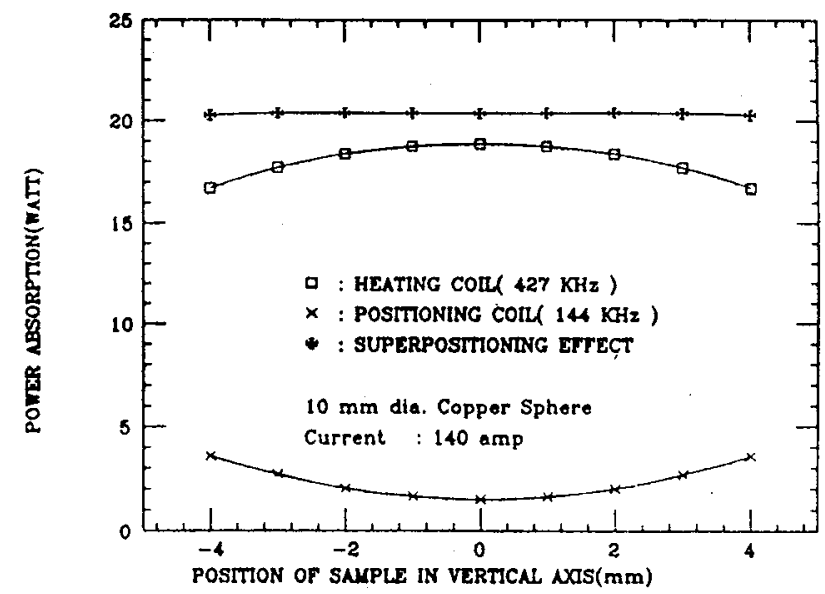

Fig. 12. Superpositioning effect of the power absorption for heating and positioning coils in TEMPUS facility.

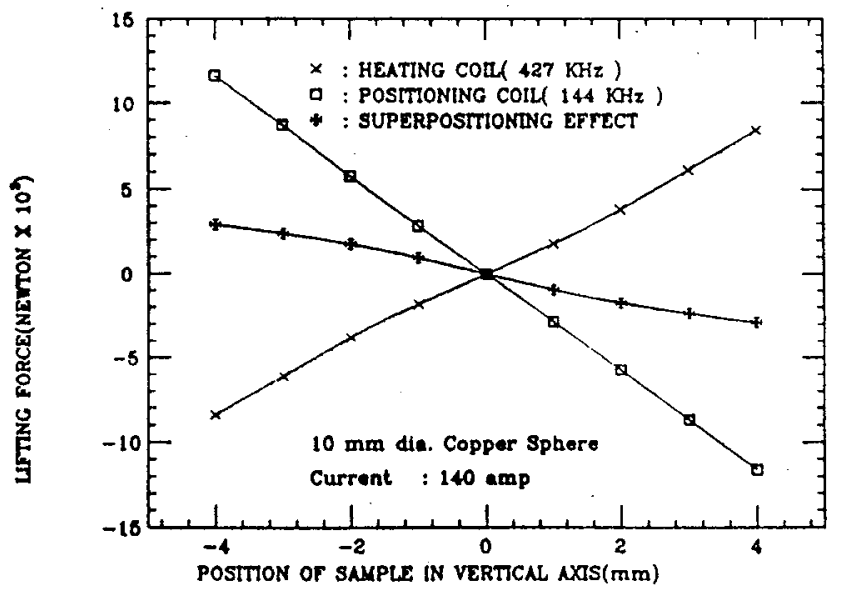

Fig. 13. Superpositioning eflect of lifting force for heating and powitioning coils in TEMPUS facility

plies that the sample can be levitated as long as the lifting force is greater than the gravitational force.

Fig. 14 shows the effect of sample size on power absorption examined with the same coil geometry as previous paper [24] with an in-phase current of $300 \mathrm{~A}$ and a 


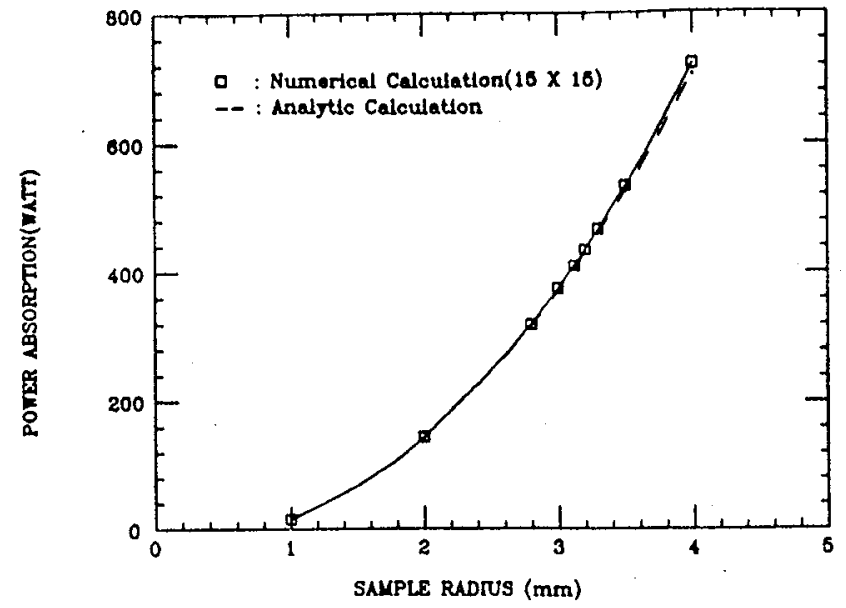

Fig. 14. Effects of sample size on power absorption (in-phase $300 \mathrm{~A}$ current by the coils of [24], $450 \mathrm{kHz}$ ).

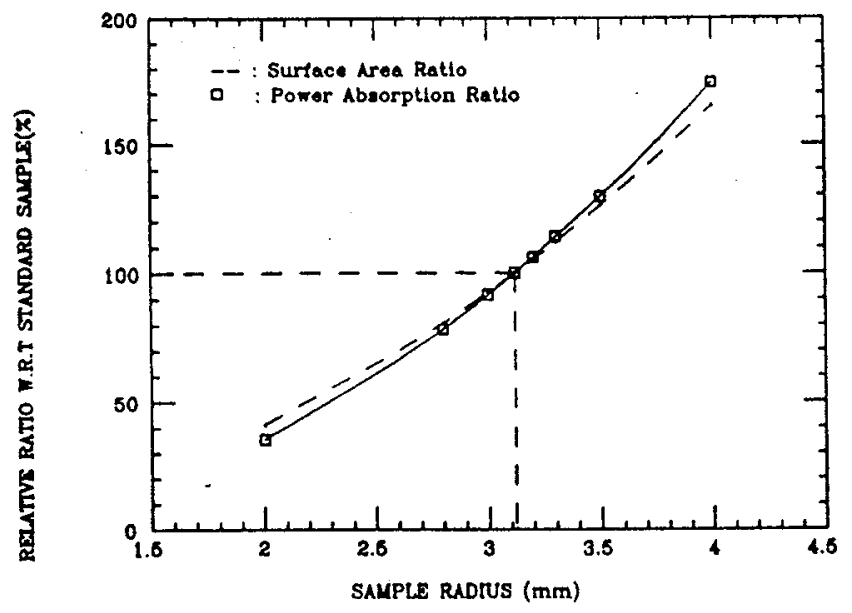

Fig. 15. Comparison of variation of power absorption and surface areal of sample with the sample size $(300 \mathrm{~A}, 450 \mathrm{kHz}$, by the coils of $|24|$. dotted line indicates standard sample of $1 \mathrm{~g}$ iron drop).

frequency of $450 \mathrm{kHz}$. As can be seen from the curve, the power absorption increases in a parabolic fashion with the sample radius. Because the surface area of the sample is also proportional to the square of the sample radius, the relationship between power absorption and surface area was examined, as illustrated in Fig. 15, with respect to the standard sample of a one gram iron drop. It shows that the power absorption is approximately proportional to the surface area. This approximation will be especially useful for the case of small skin depths.

Fig. 16 illustrates the electromagnetic calculations for a deformed niobium sample of predefined shape at $2500^{\circ} \mathrm{C}$ in a quadrupole field. In this case, the skin depth is quite large because of high temperature. The field calculations are performed quite readily, in spite of the substantial departure from the spherical shape. The computational time (about $8 \mathrm{~min}$ ) was comparable to that needed for calculations with an undeformed spherical sample. This is a major attractiveness of the technique.

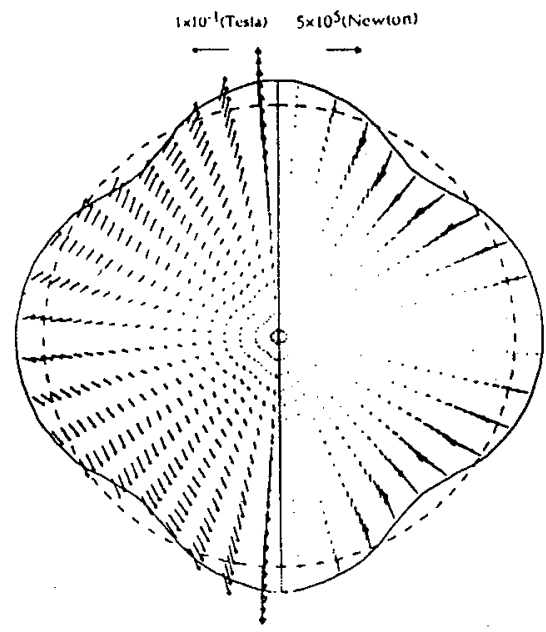

Fig. 16. Computed results of instant magnetic field density (left) and force field (right) for predefined shape by TEMPUS quadrupole field $\left(2500^{\circ} \mathrm{C}\right.$ niobium, $1400 \mathrm{~A}, 144 \mathrm{kHz}$, dotted line represents $6 \mathrm{~mm}$ dia sphere).

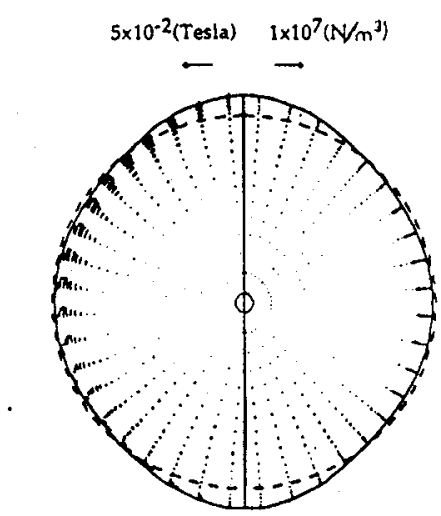

Fig. 17. Computed shape of the squeczed sample by the present electromagnetic calculation scheme (for $10 \mathrm{~mm}$ dia silver drop with $280 \mathrm{~A}, 144$ $k H z)$.

Finally, Fig. 17 shows the calculated equilibrium shape of a silver droplet which is noticeably deformed as a result of using a single coil in a microgravity setting. The iterative scheme used for these calculations was outlined earlier in this manuscript. In the present case a total of $\sim 100$ iterations was needed. We used a $15 \times 15$ grid during the iterations for shape calculation and an additional calculation was carried out by a $20 \times 20$ grid in order to obtain the final results. Hence the total computational time was about $20 \mathrm{~min}$ on a micro-VAX 3100 workstation.

This example readily illustrates the usefulness of this technique. It is of interest to note that while the deformed droplet had an increase in the surface area of $\sim 4.5 \%$, the actual heating rate was reduced by some $31.4 \%$ because the distance between the coil and the sample surface was substantially increased as a result of the deformation. This calculation is quite important in the design of levitation melting experiments because it clearly shows that deformation of the sample will markedly alter the thermal energy input. 


\section{Concluding Remarks}

An efficient computational technique has been presented to describe the electromagnetic forces and the power absorption in a levitation melted sample. In essence the technique involves the use of a volume integral coupled with a coordinate transformation, which renders the method particularly attractive for tackling problems involving substantially deformed specimens that still obey rotational symmetry.

The computed results were tested against an analytical solution for spherical specimens and the agreement was excellent, even for a relatively coarse grid structure.

The calculations were then extended to represent the behavior of nonspherical samples and to calculate the shape of a metal droplet in the presence of a strong magnetic field by means of a force balance and the iterative use of the previously described computational scheme. This task could be readily performed in a fairly efficient manner.

As a practical matter the computational scheme is being used for the rational design of levitation melting experiments in microgravity processing, but the same procedure may also be utilized for tackling a broader class of electromagnetic shaping and meniscus control problems, provided rotational symmetry is being observed.

\section{REFERENCES}

[1] S. Asai, "Recent study on electromagnetic processing of material," in Metallurgical Processes for the Year 2000 and Beyond, TMS Publication, H. Y. Shon, (ed.) 1989.

[2] A. J. Mestel. "Magnetic levitation of liquid metal," J. Fluid Mech., vol. 117. pp. 27-43, 1982.

[3] A. Gagnoud. J. Etay, and M. Garnier. "The tevitation melting process using cold crucible technique," Trans. I.S.I.J.. vol. 28. pp. 36$40,1986$.

[4] N. El-Kaddah and J. Szekely, "Heat and fluid flow phenomena in a levitation melted sphere under zero gravity condition," Met. Trans., vol. 15B, pp. 183-186, 1984.

[5] R. Willnecker, D. M. Herlach, and B. Feuerbacher, "Undercooling of bulk metals by application of electromagnetic levitation technique," Proc. 6th European Symposium on Material Sciences under Micro-gravity condition. Bordeaux. France, pp. 339-344, Dec. 1986.

[6] Technical Reports: "Description of experimental facilities and science capabilities." Containerless Experimentation in Microgravity Workshop, Jet Propulsion Laboratory. Pasadena, Jan. 1990.

[7] TEMPUS Manual: "Containerless processing under microgravity condition," published in DORNIER Post, Jan. 1987

[8] G. Lohofer, "Theory of an electromagnetically levitated metal sphere (I): Absorbed Power," SIAM J. Appl. Math., vol. 49, April, pp. 567-581. 1989.

[9] R. F. Dudley and P. E. Burke. "The prediction of current distribution in induction heating installations." IEEE Trans. Ind. Afpl. vol. IA. 8. pp. $565-571,1972$.

[10] E. D. Tarapore and J.W. Evans, "Fluid velocities in induction melting fumace, part I. Theory and Laboratory experiments" Met. Trans., vol. 7B, pp. 343-351, 1976.

[11] M. V. K. Chari and P. P. Silverster: "Finite Elements in Electric and Magneric Field Problems," New York: Wiley, 1980.

[12] A. Konrad. "Eddy Currents and Modeling." IEEE Trans. Magn.. vol. MAG-21. pp. 1805-1810, 1985 .

[13] M. H. Lean. "Application of boundary integral equation methods to electromagnetics," IEEE Trans. Magn., vol. Mag-21, pp. 18231827.

[14] M. Ramadan Ahmed, J. D. Lavers and P. E. Burke: "A coupled BEIBC formulation for multiple conductor eddy current problem." $I E E E$ Trans. Magn., vol. 24, No. 6, pp. 2524-2526. 1988

[15] J. D. Lavers, "Computational methods for the analysis of molten metal electromagnetic confinement system. " ISIJ International, vol. 29. No. 12. pp. 993-1005, 1989.

[16] J. D. Lavers and P. P. Bringer: "The influence of system geometry on the electromagnetic stirring forces in crucible induction melting furnaces," Metallurgical Applications of Magnetohydrodynamics, Metal Society, London, H. K. Moffatt and M.R.E. Proctor (eds.), 1982.

[17] T. Toh and M. Garnier: "Numerical approach to the inverse free boundary problem in the cold crucible for levitation." presented at the 7th Int. Conf. on Physico-Chemical Hydrodymumics, MIT. Cumbridge. June 1989.

[18] N. El-Kaddah and F. A. Acosta-Gonzalez, "Mathematical model for the shaping of molten metal by an electromagnetic field." Proc. of Int. Symposium on Casting of Near Net Shape Products, Honolulu, Nov. 1988.

[19] G. O. Roberts, "Computational meshes for boundary layer problem." Proc. 2nd Int. Conf. Num. Methods Fluid Dyn. Lecture Notes in Physics. vol. 8, pp. 171-177, 1971.

(20) J. F. Tompson, "General curvilinear coordinate systems," in $\mathrm{Nu}$ merical Grid Generation. J. F. Tompson (ed.), 1982

[21] A. J. Mestel: "Levitation melting of metals," Metallurgical Applications of Magnetohydrodynamics, Metal Society, London, H. K. Moffatt and M.R.E. Proctor (eds.), 1982.

[22] A. Gagnoud, J. Etay, and M. Garnier, "The problem of free boundary in electromagnetic levitation." $J$. Theoretical Appl. Mech., vol. 5. No. 6, pp. 911-934, 1986.

[23] Private communication with $G$. Lohofer.

[24] N. El-Kaddah and J. Szekely: "The electromagnetic force field, fluid flow field, and temperature profiles in levitated metal droplets. " Met. Trans.. vol. 14B, pp. 401-410, 1983.

Jin-Ho Zong, was born in Seoul, Korea, May 20, 1958. He received the B.S., M.S., and Ph.D. degrees in 1981, 1984, and 1988, respectively. from the Department of Metallurgical Engineering at Scoul National Uni. versity, Seoul. Korea.

He was a Research Associate at Seoul National University from 1985 to 1988. Now, he is a Postdoctoral Associate with the Department of Materials Science and Engineering. Massachusetts Institute of Technology. Cambridge, MA.

$\mathrm{He}$ is a member of the Korean Institute of Metals.

Julian Szekely, was born in Budapest. Hungary. November 23. 1934. He received the B.Sc. and $\mathrm{Ph} . \mathrm{D}$. degrees in 1959 and 1961, respectively, in chemical engineering from Imperial College, London.

$\mathrm{He}$ is a member of the US National Academy of Engineering, the American Institute of Mining. Metallurgical and Petroleum Engineers, the Institute of Chemical Engineers, the Metal Society, and the Japanese Iron and Steel Institute.

Before joining MIT's faculty in the Department of Material Science and Engineering in 1975, he was on the faculty of Imperial College and State University of New York at Buffalo.

Elliot Schwartz was born in Brooklyn, NY, on April 26, 1967. He received the B.S. degrees in materials science and engineering and mathematics from the Massachusetts Institute of Technology in 1989. He is currently pursuing the Ph.D. degree in the Department of Materials Science and Engineering at MIT.

$\mathrm{Hc}$ is a member of the Minerals, Metals, and Materials Society. 